PROCEEDINGS OF THE

AMERICAN MATHEMATICAL SOCIETY

Volume 135, Number 12, December 2007, Pages 3949-3954

S 0002-9939(07)09000-4

Article electronically published on September 7, 2007

\title{
AN EXAMPLE ON ORDERED BANACH ALGEBRAS
}

\author{
GERD HERZOG AND CHRISTOPH SCHMOEGER
}

(Communicated by Joseph A. Ball)

\begin{abstract}
Let $\mathcal{B}$ be a complex unital Banach algebra. We consider the Banach algebra $\mathcal{A}=\mathcal{B} \times \mathbb{C}$ ordered by the algebra cone $K=\{(a, \xi) \in \mathcal{A}:\|a\| \leq$ $\xi\}$, and investigate the connection between results on ordered Banach algebras and the right bound of the numerical range of elements in $\mathcal{B}$.
\end{abstract}

\section{ORdered BANACH ALGEBras}

The aim of this paper is to stress the aspect of the applicability of the ordered Banach algebra theory, within a wider scope of general Banach algebras. To this end we will embed a given (non-ordered) Banach algebra $\mathcal{B}$ into a certain ordered Banach algebra $\mathcal{A}$; see section 2. In particular Theorems 6 and 7 in section 5 are results strictly in terms of the originally given Banach algebra $\mathcal{B}$, while the proofs involve results on ordered Banach algebras applied to $\mathcal{A}$. We start with some notations and results on general ordered Banach algebras.

Let $\mathcal{A}$ be a complex unital Banach algebra with unit $\mathbf{1}$, and assume that $\mathcal{A}$ is ordered by an algebra cone $K$, that is, $K$ is a closed convex subset of $\mathcal{A}$ with $\lambda K \subseteq K(\lambda \geq 0), K \cap(-K)=\{0\}, \mathbf{1} \in K, K \cdot K \subseteq K$, and $a \leq b: \Longleftrightarrow b-a \in K$.

For a general survey on ordered Banach algebras we refer to 4, 9, and the references given there.

The spectrum of $a \in \mathcal{A}$ is denoted by $\sigma(a)$, and $r(a)$ denotes its spectral radius. Moreover let $\tau(a)$ denote the right spectral bound of $a$, that is,

$$
\tau(a):=\max \{\operatorname{Re} \lambda: \lambda \in \sigma(a)\} .
$$

Next, we consider the sets

$$
Q_{+}:=\{a \in \mathcal{A}: \exp (t a) \geq 0(t \geq 0)\}, \quad Q_{ \pm}=Q_{+} \cap\left(-Q_{+}\right) .
$$

Amongst other things the following properties of these sets are known. Note that $\emptyset \neq W \subseteq \mathcal{A}$ is called a wedge if $W$ is closed, convex and $\lambda W \subseteq W(\lambda \geq 0)$.

Theorem 1. Under the assumptions above

1. $Q_{+}$is a wedge, and $\overline{K+\mathbb{R} \mathbf{1}}=Q_{+}$;

2. $Q_{ \pm}$is a closed real subspace of $\mathcal{A}$;

3. $Q_{ \pm}$is a real Lie algebra;

4. $a \in Q_{ \pm} \Longrightarrow a^{2} \in Q_{+}$;

Received by the editors September 22, 2006 and, in revised form, November 6, 2006

2000 Mathematics Subject Classification. Primary 47H05, 47A12, 47B60.

Key words and phrases. Ordered Banach algebras, bounds of numerical range, fractional powers.

(C)2007 American Mathematical Society Reverts to public domain 28 years from publication 
5. $a \in Q_{+}, \tau(a)<0 \Longrightarrow a^{-1} \leq 0$;

6. $a \in Q_{+}, \tau(a)<0 \Longrightarrow a(\lambda \mathbf{1}-a)^{-1} \in Q_{+}(\lambda \geq 0)$.

Proof. Parts 1, 2, 4 and 5 of Theorem 1 have been proved in [4 for real Banach algebras (where then in part $5 \tau(a)$ is obtained by complexification), but hold obviously in the complex case by considering $\mathcal{A}$ as a real Banach algebra (by restricting the scalar field to $\mathbb{R}$ ). Part 3 of Theorem 1 follows easily from the commutator formula for the exponential function; see [5, Lemma I.2.13]. To prove part 6 note that $\tau(a-\lambda \mathbf{1})<0(\lambda \geq 0)$ and consider

$$
a(\lambda \mathbf{1}-a)^{-1}=(\lambda \mathbf{1}+a-\lambda \mathbf{1})(\lambda \mathbf{1}-a)^{-1}=-\lambda(a-\lambda \mathbf{1})^{-1}-\mathbf{1} .
$$

Thus $a(\lambda \mathbf{1}-a)^{-1} \in K+\mathbb{R} \mathbf{1}$ according to part 5 ; hence it is in $Q_{+}$by 1 .

In particular part 6 of Theorem 1 can be used to find roots of elements of $-Q_{+}$ in $Q_{+}$by using the formula of Balakrishnan [10, p.260].

Theorem 2. Let $a \in Q_{+}$with $\tau(a)<0$, and let $\alpha \in(0,1)$. Then

$$
-(-a)^{\alpha}:=\frac{\sin (\alpha \pi)}{\pi} \int_{0}^{\infty} \lambda^{\alpha-1} a(\lambda \mathbf{1}-a)^{-1} d \lambda \in Q_{+} .
$$

Proof. By means of part 6 of Theorem 1 we see that the integrand is in $Q_{+}$for each $\lambda \in(0, \infty)$. Since $Q_{+}$is a wedge we conclude that $-(-a)^{\alpha} \in Q_{+}$.

For our third theorem, which is on majorization, let the unital Banach algebra $\mathcal{A} \times \mathcal{A}$ (endowed with coordinatewise multiplication and the norm $\|(a, b)\|=$ $\max \{\|a\|,\|b\|\})$ be ordered by the algebra cone

$$
\widetilde{K}=\{(a, b) \in \mathcal{A} \times \mathcal{A}:-b \leq a \leq b\},
$$

and let $\widetilde{Q}_{+}$denote the corresponding set of exponentially nonnegative elements. The following theorem is proved in [3] for operators on ordered Banach spaces and holds with an analog proof for Banach algebras in the following version:

Theorem 3. Let $a, b \in \mathcal{A}$ be such that $a \leq b$ and $a+b \in Q_{+}$. Then $(a, b) \in \widetilde{Q}_{+}$.

\section{Special ordered BanaCh ALGEBras}

Now, let $\mathcal{B}$ be any complex unital Banach algebra with unit $\mathbf{1}$ and let $\mathcal{A}$ denote the Banach algebra $\mathcal{B} \times \mathbb{C}$, endowed with multiplication and norm

$$
(a, \xi) \cdot(b, \eta)=(a b, \xi \eta), \quad\|(a, \xi)\|=\max \{\|a\|,|\xi|\}
$$

respectively. Then $\mathcal{A}$ is a unital Banach algebra with unit $(\mathbf{1}, 1)$, and we consider the ordering defined by the cone

$$
K=\{(a, \xi) \in \mathcal{A}:\|a\| \leq \xi\}
$$

We will characterize $Q_{+}$and $Q_{ \pm}$in this case, and we will obtain results on the right bound of the numerical range of elements of $\mathcal{B}$, by means of Theorem 1 . 


\section{BOUNDS OF THE NUMERICAL RANGE}

Let $\mathcal{B}$ be a complex unital Banach algebra and consider the one-sided directional derivatives of the norm at $\mathbf{1}$ :

$$
n_{+}[a]=\lim _{h \rightarrow 0+} \frac{\|\mathbf{1}+h a\|-1}{h}, \quad n_{-}[a]=\lim _{h \rightarrow 0-} \frac{\|\mathbf{1}+h a\|-1}{h} \quad(a \in \mathcal{B}) .
$$

For each $a \in \mathcal{B}$ let $V(a)$ denote the numerical range of $a$, that is,

$$
V(a)=\left\{\varphi(a): \varphi \in \mathcal{B}^{*},\|\varphi\|=\varphi(\mathbf{1})=1\right\} .
$$

The set $V(a)$ is bounded closed and convex, and $\sigma(a) \subseteq V(a)$ for each $a \in \mathcal{B}$; see 2]. The number $n_{+}[a]$ is the right bound of the numerical range of $a$, that is,

$$
n_{+}[a]=\max \{\operatorname{Re} \lambda: \lambda \in V(a)\},
$$

according to Mazur's representation of directional derivatives of sublinear functionals [8]; see also [2], 7]. In particular $\tau(a) \leq n_{+}[a]$.

Note the following properties of $n_{+}$and $n_{-}$: The function $a \mapsto n_{+}[a]$ is sublinear, and

$$
n_{-}[a] \leq n_{+}[a], \quad n_{-}[-a]=-n_{+}[a], \quad n_{ \pm}[a+\lambda \mathbf{1}]=n_{ \pm}[a]+\lambda
$$

for all $a \in \mathcal{B}$ and $\lambda \in \mathbb{R}$.

Next, let $H(\mathcal{B})$ denote the set of all hermitian elements of $\mathcal{B}$, that is,

$$
H(\mathcal{B})=\{a \in \mathcal{B}:\|\exp (i t a)\|=1(t \in \mathbb{R})\} .
$$

For the following properties of hermitian elements see [2, 5. Lemma 2] for part 1 and [1] for part 2.

\section{Proposition 1. We have}

1. $a \in H(\mathcal{B}) \Longleftrightarrow V(a) \subseteq \mathbb{R} \Longleftrightarrow n_{+}[i a]=n_{-}[i a]=0$;

2. $a \in H(\mathcal{B}) \Longleftrightarrow \sigma(a) \subseteq \mathbb{R}$ and $r(\alpha \mathbf{1}+\beta a)=\|\alpha \mathbf{1}+\beta a\|(\alpha, \beta \in \mathbb{C})$.

Now, in the sequel let $\mathcal{A}=\mathcal{B} \times \mathbb{C}$ be as in section 2 .

\section{Representation of $Q_{+}$And $Q_{ \pm}$}

Note that

$$
Q_{+}=\{(a, \xi) \in \mathcal{A}:\|\exp (t a)\| \leq \exp (t \xi)(t \geq 0)\}
$$

in our case.

Theorem 4. We have

1. $(a, \xi) \in Q_{+}$if and only if $n_{+}[a] \leq \xi$.

2. $(a, \xi) \in Q_{ \pm}$if and only if one of the following equivalent conditions is valid:

(a) $\|\exp (t a)\|=\exp (t \xi)(t \in \mathbb{R})$;

(b) $n_{+}[a]=n_{-}[a]=\xi$;

(c) $\xi \in \mathbb{R}$ and $i(a-\xi \mathbf{1}) \in H(\mathcal{B})$;

(d) $\operatorname{Re} \lambda=\xi(\lambda \in \sigma(a))$ and $r(\alpha \mathbf{1}+\beta a)=\|\alpha \mathbf{1}+\beta a\|(\alpha, \beta \in \mathbb{C})$.

Proof. 1. First note that for each $a \in \mathcal{B}$

$$
\|\exp (t a)\| \leq \exp \left(t n_{+}[a]\right) \quad(t \geq 0) ;
$$

see [2, 3. Theorem 4]. Thus, $n_{+}[a] \leq \xi$ implies $(a, \xi) \in Q_{+}$. On the other hand, $(a, \xi) \in Q_{+}$implies

$$
\frac{\|\exp (t a)\|-1}{t} \leq \frac{\exp (t \xi)-1}{t} \quad(t>0)
$$


and as $t \rightarrow 0+$ we obtain $n_{+}[a] \leq \xi$, since $\exp (t a)=1+t a+O\left(t^{2}\right)(t \rightarrow 0+)$.

2. Let $(a, \xi) \in Q_{ \pm}$. Then $\|\exp (t a)\| \leq \exp (t \xi)(t \in \mathbb{R})$. From

$$
\|\exp (t a)\| \geq \frac{1}{\left\|(\exp (t a))^{-1}\right\|}=\frac{1}{\|\exp (-t a)\|} \geq \frac{1}{\exp (-t \xi)}=\exp (t \xi)
$$

we obtain $\|\exp (t a)\|=\exp (t \xi)(t \in \mathbb{R})$.

Next, by means of part 1 we have

$$
n_{-}[a] \leq n_{+}[a] \leq \xi,-n_{-}[a]=n_{+}[-a] \leq-\xi,
$$

thus

$$
\xi \leq n_{-}[a] \leq n_{+}[a] \leq \xi \Rightarrow n_{-}[a]=n_{+}[a]=\xi .
$$

In particular, $\xi \in \mathbb{R}$ and

$$
n_{ \pm}[a-\xi \mathbf{1}]=n_{ \pm}[a]-\xi=0 .
$$

Thus

$$
\lim _{h \rightarrow 0} \frac{\|1+h(a-\xi \mathbf{1})\|-1}{h}=0,
$$

and according to part 1 of Proposition $1, i(a-\xi \mathbf{1}) \in H(\mathcal{B})$.

Since clearly each element $(a, \xi)$ with the property in (a) or (b) is in $Q_{ \pm}$and since (c) implies (b) according to part 1 of Proposition 1 , we have proved that $(a, \xi) \in Q_{ \pm}$if and only if $(a, \xi)$ satisfies (a), (b) or (c), respectively

To prove the equivalence of (c) and (d) first let $(a, \xi)$ be such that $\xi \in \mathbb{R}$ and $b:=i(a-\xi \mathbf{1}) \in H(\mathcal{B})$. According to part 2 of Proposition 1

$$
\sigma(b) \subseteq \mathbb{R} \Rightarrow \sigma(a) \subseteq\{\xi+i t: t \in \mathbb{R}\} .
$$

For $\alpha, \beta \in \mathbb{C}$ we obtain

$$
r(\alpha \mathbf{1}+\beta a)=r((\alpha+\xi \beta) \mathbf{1}-i \beta b)=\|(\alpha+\xi \beta) \mathbf{1}-i \beta b\|=\|\alpha \mathbf{1}+\beta a\| .
$$

Vice versa, let $(a, \xi)$ satisfy $(\mathrm{d})$, and set $b:=i(a-\xi \mathbf{1})$. Then $\xi \in \mathbb{R}$, and $\sigma(b) \subseteq \mathbb{R}$. For $\alpha, \beta \in \mathbb{C}$ we have

$$
\alpha \mathbf{1}+\beta b=(\alpha-i \xi \beta) \mathbf{1}+i \beta a,
$$

thus

$$
r(\alpha \mathbf{1}+\beta b)=\|\alpha \mathbf{1}+\beta b\| .
$$

Once more using part 2 of Proposition 1 we conclude $b \in H(\mathcal{B})$.

\section{Applications}

By application of Theorem 1 to the algebra $\mathcal{A}$ we obtain:

Theorem 5. Let $a, b \in \mathcal{B}$.

1. If $a, b \in H(\mathcal{B})$, then $i(a b-b a) \in H(\mathcal{B})$; compare [2, 5. Lemma 4].

2. If $a \in H(\mathcal{B})$, then $n_{+}\left[-a^{2}\right] \leq 0$.

3. $\tau\left(\left(a, n_{+}[a]\right)\right)=n_{+}[a]$.

4. If $n_{+}[a]<0$, then $\left\|a^{-1}\right\| \leq-\left(n_{+}[a]\right)^{-1}$; compare [6. Proposition1.1(3)].

5. If $n_{+}[a]<0$, then

$$
n_{+}\left[a(\lambda \mathbf{1}-a)^{-1}\right] \leq \frac{n_{+}[a]}{\lambda-n_{+}[a]} \quad(\lambda \geq 0) .
$$


Proof. 1. According to Theorem 4 we have $(i a, 0),(i b, 0) \in Q_{ \pm}$. Hence $(-a b+$ $b a, 0) \in Q_{ \pm}$, and again Theorem 4 proves $i(a b-b a) \in H(\mathcal{B})$.

2. Since $(i a, 0) \in Q_{ \pm}$we have $\left(-a^{2}, 0\right) \in Q_{+}$. Hence $n_{+}\left[-a^{2}\right] \leq 0$.

3. If $\operatorname{Re} \lambda>n_{+}[a]$, then $\left(a, n_{+}[a]\right)-\lambda(\mathbf{1}, 1)$ is invertible, since $\tau(a) \leq n_{+}[a]$ and $n_{+}[a]-\lambda \neq 0$. Moreover $\left(a, n_{+}[a]\right)-n_{+}[a](\mathbf{1}, 1)=\left(a-n_{+}[a] \mathbf{1}, 0\right)$ is not invertible. Thus $n_{+}[a] \in \sigma\left(\left(a, n_{+}[a]\right)\right)$.

4. We have $\left(a, n_{+}[a]\right) \in Q_{+}$, and $\tau\left(\left(a, n_{+}[a]\right)\right)=n_{+}[a]<0$. Thus

$$
-\left(a, n_{+}[a]\right)^{-1}=\left(-a^{-1},\left(-n_{+}[a]\right)^{-1}\right) \geq 0,
$$

which means $\left\|a^{-1}\right\| \leq-\left(n_{+}[a]\right)^{-1}$.

5. Since $\left(a, n_{+}[a]\right) \in Q_{+}$and since $\tau\left(\left(a, n_{+}[a]\right)\right)=n_{+}[a]<0$ we have

$$
\begin{gathered}
\left(a, n_{+}[a]\right)\left(\lambda(\mathbf{1}, 1)-\left(a, n_{+}[a]\right)\right)^{-1} \\
=\left(a(\lambda \mathbf{1}-a)^{-1}, n_{+}[a]\left(\lambda-n_{+}[a]\right)^{-1}\right) \in Q_{+}
\end{gathered}
$$

for each $\lambda \geq 0$. According to Theorem 4

$$
n_{+}\left[a(\lambda \mathbf{1}-a)^{-1}\right] \leq \frac{n_{+}[a]}{\lambda-n_{+}[a]} \quad(\lambda \geq 0) .
$$

Analogously we can apply Theorem 2 and derive one sided estimates for fractional powers:

Theorem 6. Let $a \in \mathcal{B}$ with $n_{+}[a]<0$, and $\alpha \in(0,1)$. Then

$$
n_{+}\left[-(-a)^{\alpha}\right] \leq-\left(-n_{+}[a]\right)^{\alpha} .
$$

Proof. Since $\left(a, n_{+}[a]\right) \in Q_{+}$we have

$$
-\left(-\left(a, n_{+}[a]\right)\right)^{\alpha}=\left(-(-a)^{\alpha},-\left(-n_{+}[a]\right)^{\alpha}\right) \in Q_{+} .
$$

Remark. For results on the numerical range of roots in Banach algebras see [6] and the references given there.

Next, Theorem 3 leads to the following inequalities for the exponential function in Banach algebras:

Theorem 7. Let $a, b \in \mathcal{B}$. Then

$$
\|\exp (b)-\exp (a)\| \leq 2 \sinh \left(\frac{\|b-a\|}{2}\right) \exp \left(\frac{n_{+}[a+b]}{2}\right)
$$

and

$$
\|\exp (b)+\exp (a)\| \leq 2 \cosh \left(\frac{\|b-a\|}{2}\right) \exp \left(\frac{n_{+}[a+b]}{2}\right) .
$$

Proof. We set

$$
\alpha=\frac{n_{+}[a+b]-\|b-a\|}{2}, \quad \beta=\frac{n_{+}[a+b]+\|b-a\|}{2} .
$$

Then

$$
\|b-a\|=\beta-\alpha \Rightarrow(a, \alpha) \leq(b, \beta)
$$

and

$$
n_{+}[a+b]=\alpha+\beta \Rightarrow(a, \alpha)+(b, \beta) \in Q_{+} .
$$


According to Theorem 3 we have $((a, \alpha),(b, \beta)) \in \widetilde{Q}_{+}$. Hence

$$
-(\exp (t b), \exp (t \beta)) \leq(\exp (t a), \exp (t \alpha)) \leq(\exp (t b), \exp (t \beta)) \quad(t \geq 0)
$$

For $t=1$ we obtain

$$
\|\exp (b)-\exp (a)\| \leq \exp (\beta)-\exp (\alpha)=2 \sinh \left(\frac{\|b-a\|}{2}\right) \exp \left(\frac{n_{+}[a+b]}{2}\right),
$$

and the second inequality in an analog way.

Remark. The inequalities in Theorem 7 are quite obvious if $a$ and $b$ commute.

Then, for example,

$$
\begin{gathered}
\|\exp (b)-\exp (a)\|=\left\|2 \sinh \left(\frac{b-a}{2}\right) \exp \left(\frac{a+b}{2}\right)\right\| \\
\leq 2 \sinh \left(\frac{\|b-a\|}{2}\right)\left\|\exp \left(\frac{a+b}{2}\right)\right\| \leq 2 \sinh \left(\frac{\|b-a\|}{2}\right) \exp \left(\frac{n_{+}[a+b]}{2}\right) .
\end{gathered}
$$

\section{REFERENCES}

[1] Baillet, M.: Sur les éléments hermitiens d'une algèbre de Banach et les dilatations de certains opérateurs normaloides. C. R. Acad. Sci., Paris, Ser. A 281, 1039-1042 (1975). MR0390822 $(52: 11645)$

[2] Bonsall, F.F.; Duncan, J.: Numerical ranges of operators on normed spaces and of elements of normed algebras. London: Cambridge University Press. II (1971). MR0288583 (44:5779)

[3] Herzog, G.; Kunstmann, P.C.: Majorization of $C_{0}$-semigroups in ordered Banach spaces. Comment. Math. Univ. Carolin. 47, 47-54 (2006). MR2223966

[4] Herzog, G.; Lemmert, R.: On quasipositive elements in ordered Banach algebras. Stud. Math. 129, 59-65 (1998). MR1611855 (99g:46061)

[5] Hilgert, J.; Neeb, K.-H.: Lie-Gruppen und Lie-Algebren. Braunschweig: Vieweg (1991).

[6] Li, C.-K.; Rodman, L.; Spitkovsky, I.M.: On numerical ranges and roots. J. Math. Anal. Appl. 282, 329-340 (2003). MR2000347 (2004g:47009)

[7] Martin, R.H.: Nonlinear operators and differential equations in Banach spaces. Pure and Applied Mathematics. New York etc.: John Wiley\&Sons XI (1976). MR0492671 (58:11753)

[8] Mazur, S.: Über konvexe Mengen in linearen normierten Räumen. Stud. Math. 4, 70-84 (1933).

[9] Raubenheimer, H.; Rode, S.: Cones in Banach algebras. Indag. Math., New Ser. 7, 489-502 (1996). MR:1620116 (99i:46035)

[10] Yosida, K.: Functional analysis. Berlin-Göttingen-Heidelberg: Springer-Verlag. XI (1965).

Institut für Analysis, Universität Karlsruhe, D-76128 Karlsruhe, Germany

E-mail address: Gerd.Herzog@math.uni-karlsruhe.de

Institut für Analysis, Universität Karlsruhe, D-76128 Karlsruhe, Germany

E-mail address: christoph.schmoeger@math.uni-karlsruhe.de 